\title{
Fasciola hepatica: Infection Status of Freshwater Snails Collected from Gangwon-do (Province), Korea
}

\author{
Jae-Hyung Lee ${ }^{1,2, \dagger}$, Juan-Hua Quan ${ }^{3, \dagger}$, In-Wook Choi², Gab-Man Park', Guang-Ho Cha², Hyun-Ju Kim ${ }^{5}$, \\ Jae-Min Yuk', Young-Ha Lee ${ }^{2 * *}$ \\ ${ }^{1}$ Department of Medical Science, Chungnam National University Graduate School, ${ }^{2}$ Department of Infection Biology, Chungnam National University \\ College of Medicine, Daejeon 35015, Korea; ${ }^{3}$ Department of Gastroenterology, The Affiliated Hospital of Guangdong Medical College, Zhanjiang \\ 524-001, Guangdong, China; ${ }^{4}$ Department of Parasitology, Catholic Kwandong University College of Medicine, Gangneung 25601, Korea; \\ ${ }^{5}$ Rural Development Administration, Wanju-gun 55365, Korea
}

\begin{abstract}
Fasciola hepatica is a trematode that causes zoonosis, mainly in cattle and sheep, and occasionally in humans. Few recent studies have determined the infection status of this fluke in Korea. In August 2015, we collected 402 samples of freshwater snails at Hoenggye-ri (upper stream) and Suha-ri (lower stream) of Song-cheon (stream) in Daegwalnyeongmyeon, Pyeongchang-gun in Gangwon-do (Province) near many large cattle or sheep farms. F. hepatica infection was determined using PCR on the nuclear ribosomal internal transcribed spacer 2 (ITS-2). Among the 402 samples, F. hepatica 1TS-2 marker was detected in 6 freshwater snails; thus, the overall prevalence in freshwater snails was $1.5 \%$. The prevalence varied between collection areas, ranging from $0.0 \%$ at Hoenggye-ri to $2.9 \%$ at Suha-ri. However, F. gigantica ITS-2 was not detected in the 6 F. hepatica-positive samples by PCR. The nucleotide sequences of the 6 F. hepatica ITS2 PCR-positive samples were $99.4 \%$ identical to the F. hepatica ITS-2 sequences in GenBank, whereas they were $98.4 \%$ similar to $F$. gigantica ITS-2 sequences. These results indicated that the prevalence of $F$. hepatica in snail intermediate hosts was $1.5 \%$ in Gangwon-do, Korea; however the prevalence varied between collection areas. These results may help us to understand $F$. hepatica infection status in natural environments.
\end{abstract}

Key words: Fasciola hepatica, freshwater snail, Korea, PCR, ITS-2, DNA sequencing analysis

The sheep liver fluke, Fasciola hepatica, is primarily a zoonotic parasite that causes liver infection in cattle and sheep, and occasionally in humans. Freshwater snails are the first intermediate host, and humans are infected mainly by ingesting raw aquatic plants contaminated with the metacercariae $[1,2]$. F. hepatica infection in snails has traditionally been evaluated by microscopy; however, this method is time-consuming and laborious [2]. In addition, microscopic investigation of snails for F. hepatica larvae has low diagnostic sensitivity [3]. Thus, various molecular approaches have been used to identify Fasciola sp. infectons [3-9].

F. hepatica is distributed mainly in Europe, America, and Oceania [1,2]. The prevalence of $F$. hepatica infection was high in cattle before 2000 in Korea [10]. There were also several human F. hepatica infection cases [6]. However, little information

• Received 19 December 2016, revised 2 January 2017, accepted 15 January 2017. *Corresponding author (yhalee@cnu.ac.kr)

† Jae-Hyung Lee and Juan-Hua Quan contributed equally to this work.

(c) 2017, Korean Society for Parasitology and Tropical Medicine

This is an Open Access article distributed under the terms of the Creative Commons Attribution Non-Commercial License (http://creativecommons.org/licenses/by-nc/4.0) which permits unrestricted non-commercial use, distribution, and reproduction in any medium, provided the original work is properly cited. is available regarding the prevalence of $F$. hepatica infection in Korea. Therefore, to obtain basic information regarding $F$. hepatica infection in snail intermediate host in freshwater environments in Korea, we collected freshwater snails from Gangwon-do (Province) and evaluated F. hepatica contamination by PCR.

Freshwater lymnaeid snails were collected at Hoenggye-ri (upper stream) and Suha-ri (lower stream) of Song-cheon (stream) in Daegwalnyeong-myeon, Pyeongchang-gun in Gangwon-do (Province) near large cattle or sheep farms in August 2015 (Fig. 1). A total of 402 samples were collected and examined for the presence of the F. hepatica ITS-2 gene by PCR. Briefly, genomic DNA was extracted from the whole snail body using a G-DEX ${ }^{\mathrm{TM}}$ genomic DNA extraction kit (iNtRON Biotechnology, Seoul, Korea) according to the manufacturer's instructions. Genomic DNA isolated from an adult $F$. hepatica worm (kindly provided by Prof. Sung-Jong Hong, Chung-Ang University) and adult Fasciola gigantica worm (kindly provided by Prof. Keeseon S. Eom, Chungbuk National University) were used as positive controls. The sequences of the primers used were as follows: F. hepatica ITS-2, forward; GTTATAAACTAT- 


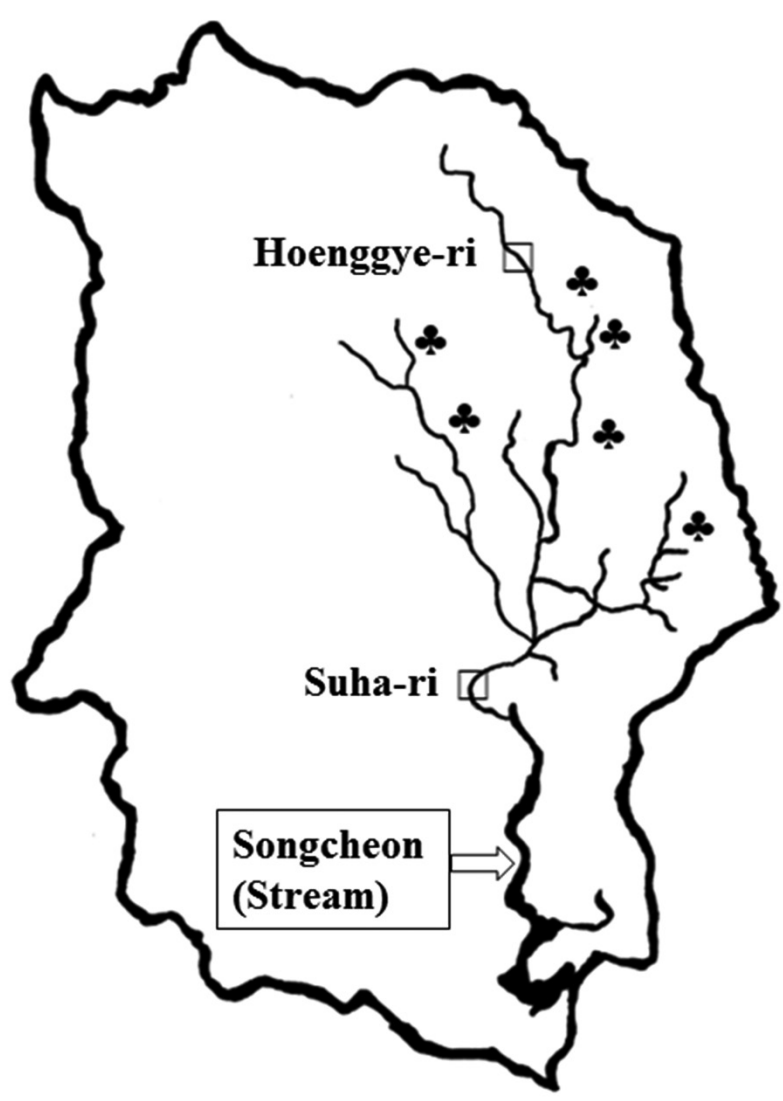

Fig. 1. Map of Daegwalnyeong-myeon, Pyeongchang-gun of Gangwon-do (Province). $\square$ Snail collected areas; Hoenggye-ri (upper stream) and Suha-ri (lower stream) of Song-cheon (stream). Large cattle or sheep farms near Song-cheon (stream) of Daegwalnyeong-myeon.

CACGACGCCCAAA, reverse; GAAGACAGACCACGAAGGGTA and $F$. gigantica ITS-2, forward; TATCACGACGCCCAAAAAGT, reverse; CCAAGTTCAGCATCAAACCA. The PCR mixture for amplification contained $5 \mu \mathrm{l}$ of genomic DNA, $3 \mu \mathrm{l}$ each of forward and reverse primers, $4 \mu \mathrm{l}$ of dNTPs, $5 \mu$ l of $10 \times$ Ex Taq buffer, $0.25 \mu$ of Ex Taq polymerase, and $29.75 \mu$ l of doubledistilled water (DDW). PCR assays were performed with an initial denaturation step of $94^{\circ} \mathrm{C}$ for $30 \mathrm{sec}$, followed by $30 \mathrm{cy}$ cles of denaturation at $98^{\circ} \mathrm{C}$ for $10 \mathrm{sec}$, annealing at $60^{\circ} \mathrm{C}$ for $30 \mathrm{sec}$, and extension at $72^{\circ} \mathrm{C}$ for $30 \mathrm{sec}$ followed by 1 cycle of $72^{\circ} \mathrm{C}$ for $10 \mathrm{~min}$ and a final hold at $4^{\circ} \mathrm{C}$. Amplification was performed using a TaKaRa PCR Thermal Cycler (Takara Bio Inc., Otsu, Japan). Agarose gel electrophoresis (1.5\%) with ethidium bromide staining was used to visualize the ITS-2 PCR products.

In addition, we confirmed the sequence of PCR products from $F$. hepatica-positive samples by DNA sequencing analysis.
A

F. hepatica ITS-2

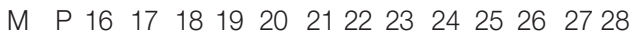

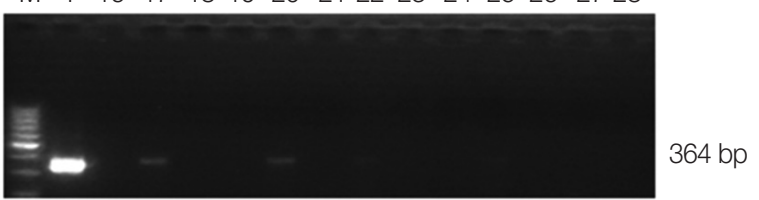

M $\quad$ P $2 \begin{array}{llllllllllll}29 & 30 & 31 & 32 & 33 & 34 & 35 & 36 & 37 & 38 & 39 & 40\end{array}$

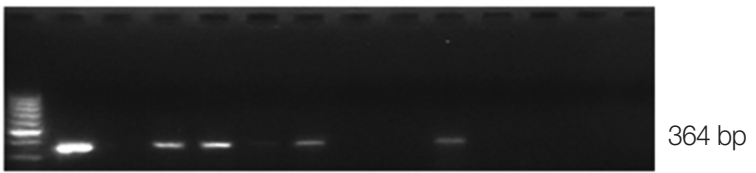

B

F. gigantica ITS-2

$\begin{array}{llllllllll}\text { M } & \mathrm{P} & 17 & 20 & 30 & 31 & 33 & 36 & 39 & 40\end{array}$

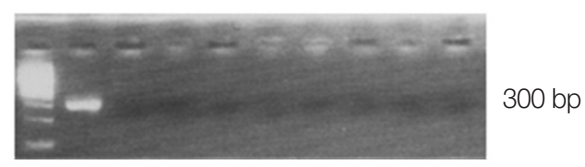

Fig. 2. Agarose gel electrophoresis of PCR products, containing the nuclear ribosomal internal transcribed spacer 2 (ITS-2) markers of Fasciola hepatica and Fasciola gigantica. (A) M, 100 bp marker; P, positive control (adult F. hepatica worm); no. 16-40, freshwater snail samples. The total length of the $F$. hepatica ITS-2 region is 364 bp. (B) M, 100 bp marker; $P$, positive control (adult $F$. gigantica worm); no. 17, 20, 30, 31, 33, and 36, F. hepatica ITS2 PCR positive samples; no. 39-40, F. hepatica ITS-2 PCR positive samples. The total length of the $F$. gigantica ITS-2 region is $300 \mathrm{bp}$.

Briefly, after electrophoretic separation, the ITS-2 PCR products were clearly delineated and sequenced directly by SolGent (Daejeon, Korea). The sequences of PCR products were compared with the complete ITS-2 sequences of F. hepatica and F. gigantica obtained from GenBank (accession nos. AJ272053.1 and EU260059.1, respectively), using Clone Manager software (Sci-Ed Software, Cary, North Carolina, USA).

PCR amplification of the positive control (adult $F$. hepatica DNA) showed a characteristic band at 364 bp for ITS-2. As shown in Fig. 2, the ITS-2 band of F. hepatica was detected in 6 of 402 snails collected from 3 areas, representing an overall $F$. hepatica infection prevalence of 1.5\% (range, 0.0-2.9\%); all were dextral. We also performed PCR analysis to evaluate F. gigantica contamination of the 6 F. hepatica ITS-2 PCR-positive samples using F. gigantica ITS-2 gene primers. After PCR, the F. gigantica ITS-2 marker was not detected among the $6 \mathrm{~F}$. hepatica-positive samples. To confirm whether the positive PCR products were from $F$. hepatica, the complete DNA sequences of the ITS-2 PCR products were compared with that in Gen- 


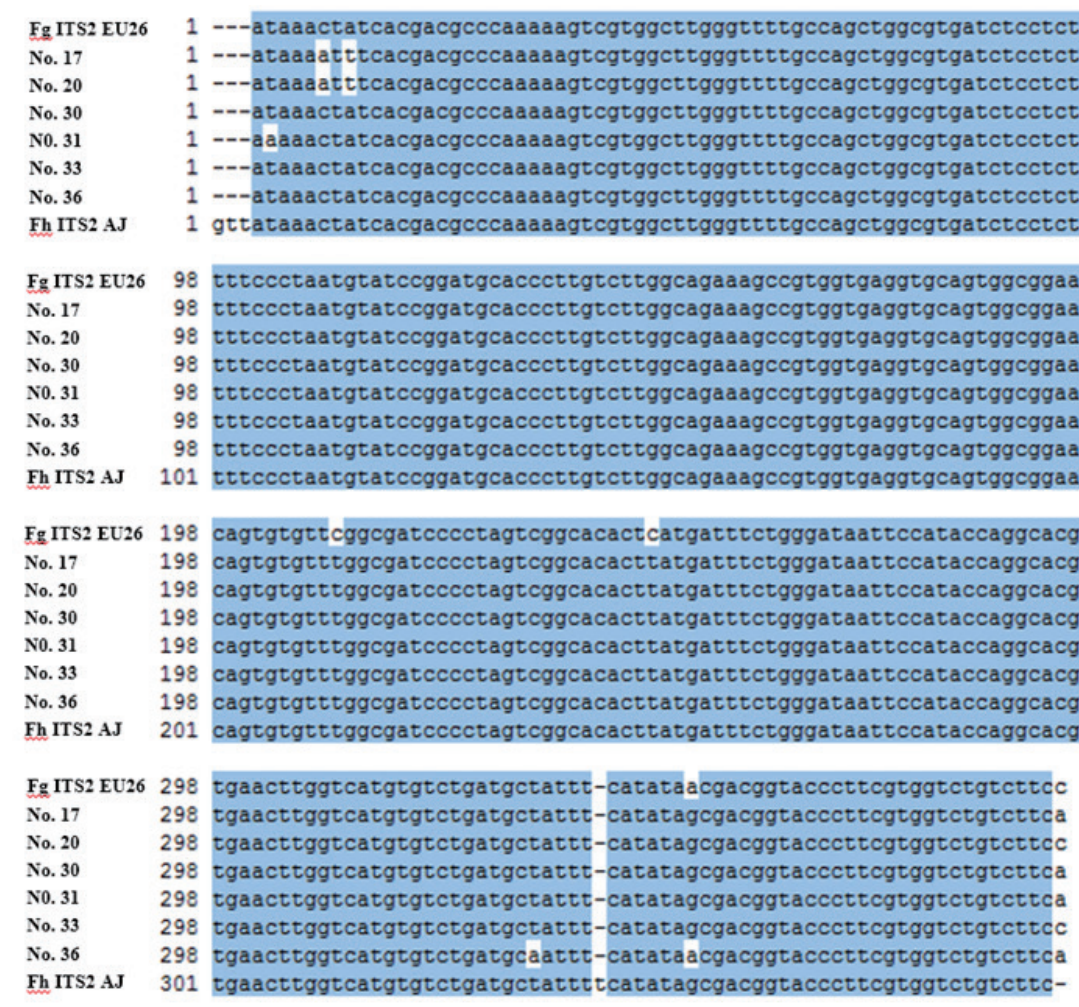

Fig. 3. The nucleotide sequences of $F$. hepatica ITS-2 PCR-positive samples were compared with the $F$. gigantica ITS-2 (accession no. EU260059.1) and F. hepatica ITS-2 genes (no. AJ272053.1) in GenBank. Base homologies are indicated by a dot (·). Fg ITS2 Eu26, F. gigantica ITS-2 GenBank sequence (no. EU260059.1); no. 17, 20, 30, 31, 33, and 36, F. hepatica ITS-2 PCR-positive samples; Fh ITS2 AJ, F. hepatica ITS-2 GenBank sequence (no. AJ272053.1).

Bank (Fig. 3). The results indicated 98.3\% similarity among 361 nucleotides between F. hepatica and F. gigantica ITS-2 sequences. The sequences of sample nos. 17, 20, 30, 31, 33, and 36 were 98.1\%, 98.1\%, 98.6\%, 98.3\%, 98.1\%, 98.6\%, and $98.6 \%$ similar to the F. gigantica ITS-2 sequence in GenBank (no. EU260059.1), respectively. However, the sequences of sample nos. 17, 20, 30, 31, 33, and 36 showed 99.2\%, 99.2\%, $99.7 \%, 99.4 \%, 99.7 \%$, and 99.2\% identity with the F. hepatica ITS-2 sequence in GenBank (no. AJ272053), respectively. Thus, the nucleotide sequences of the ITS-2 PCR products from the 6 F. hepatica PCR-positive samples were 99.4\% identical to the F. hepatica ITS-2 sequence in GenBank, but $98.4 \%$ similar to the F. gigantica ITS-2 sequences in GenBank. Taken together, the results indicated an overall prevalence of $F$. hepatica infection in freshwater snails of $1.5 \%$, ranging from $0.0 \%$ to $2.9 \%$ depending on the collection site (Table 1).

F. hepatica is a snail-transmitted trematode. Approximately 20 species within the family Lymnaeidae play an essential role as the snail intermediate host of $F$. hepatica worldwide [1,2]. In Korea, lymnaeid snails are distributed throughout the environ-
Table 1. Detection of ITS-2 genes of $F$. hepatica or F. gigantica from freshwater snails by PCR

\begin{tabular}{lccc}
\hline Areas & No. of snail & \multicolumn{2}{c}{ No. of PCR positive samples (\%) } \\
\cline { 3 - 4 } & samples & F. hepatica & F. gigantica \\
\hline $\begin{array}{c}\text { Hoenggye-ri of } \\
\text { Song-cheon (stream) }\end{array}$ & 194 & $0(0.0)$ & - \\
$\begin{array}{c}\text { Suha-ri of Song-cheon } \\
\text { (stream) }\end{array}$ & 208 & $6(2.9)$ & $0(0.0)$ \\
Total & 402 & $6(1.5)$ & $0(0.0)$ \\
\hline
\end{tabular}

ment; the major habitats are rice paddies, followed by brooks, irrigation canals, and drains [11]. In this study, we used the repetitive DNA sequence of the ITS-2 region specific for $F$. hepatica to monitor the prevalence of $F$. hepatica infection. The ITS-2 sequence of nuclear ribosomal DNA was used to identify and determine the genetic variation of $F$. hepatica $[7,9,12-14]$.

At Daegwalnyeong-myeon of PyeongChang-gun in Gangwon-do, there are many large cattle farms due to well cultivated-grass on large plateau with relatively low temperature. Thus, it would be possible to maintain the life cycle of $F$. hepatica ecologically. In the present study, we found 6 F. hepatica-infect- 
ed freshwater snails among 402 snails (1.5\%), and all $F$. hepatica-infected snails were collected at Suha-ri (lower stream), which is the lower part of many cattle or sheep farms, and thus it may be possible to be contaminated with feces of cattle. However, F. hepatica-infected snails were not found at Hoenggye-ri (upper stream), which is located at the upper part of large cattle or sheep farms. The current infection status (1.5\%) was lower than the previously reported values of 3.4\% in Korea [8], 13.0\% in Sweden [12], 7.0\% in Switzerland [4], and $26.6 \%$ in Poland [5]. In France, the prevalence of natural $F$. hepatica infection was $1.1 \%$ for Lymnaea truncatula and $0.3 \%$ for Lymnaea glabra [14].

In addition, to confirm the 6 F. hepatica ITS-2 PCR-positive samples, we performed DNA sequence analysis and compared the results with those in GenBank. The concordance rate of DNA sequences was $99.2-99.7 \%$ between the $6 \mathrm{~F}$. hepatica ITS2 PCR-positive samples and $F$. hepatica ITS- 2 sequences in GenBank. The discrepancy may have been due to point mutation of the ITS-2 gene sequences of $F$. hepatica in each snail, because the ITS-2 gene was detected in an adult $F$. hepatica worm as a positive control. In this study, the overall prevalence of $F$. hepatica infection in freshwater snails was 1.5\%, but varied according to locality. These results may help us to understand the F. hepatica infection status of natural environments.

\section{ACKNOWLEDGMENT}

The present research has been performed according to the support of Rural Development Administration, Republic of Korea (project no. PJ0108592015).

\section{CONFLICT OF INTEREST}

We have no conflict of interest related to this work.

\section{REFERENCES}

1. Mas-Coma S, Bargues MD, Valero MA. Fascioliasis and other plant-borne trematode zoonoses. Int J Parasitol 2005; 35: 1255-
1278.

2. Sripa B. Global burden of food-borne trematodiasis. Lancet Infect Dis 2012; 12:171-172.

3. Kaplan RM, Dame JB, Reddy GR, Courtney CH. The prevalence of Fasciola hepatica in its snail intermediate host determined by DNA probe assay. Int J Parasitol 1997; 27: 1585-1593.

4. Schweizer G, Meli ML, Torgerson PR, Lutz H, Deplazes P, Braun U. Prevalence of Fasciola hepatica in the intermediate host Lymnaea truncatula detected by real time TaqMan PCR in populations from 70 Swiss farms with cattle husbandry. Vet Parasitol 2007; 150: 164-169.

5. Kozak M, Wedrychowicz H. The performance of a PCR assay for field studies on the prevalence of Fasciola hepatica infection in Galba truncatula intermediate host snails. Vet Parasitol 2010; 168: 25-30.

6. Kang BK, Jung BK, Lee YS, Hwang IK, Lim H, Cho J, Hwang JH, Chai JY. A case of Fasciola hepatica infection mimicking cholangiocarcinoma and ITS-1 sequencing of the worm. Korean J Parasitol 2014; 52: 193-196.

7. Choe SE, Nguyen TT, Kang TG, Kweon CH, Kang SW. Genetic analysis of Fasciola isolates from cattle in Korea based on second internal transcribed spacer (ITS-2) sequence of nuclear ribosomal DNA. Parasitol Res 2011; 109: 833-839.

8. Kim HY, Choi IW, Kim YR, Quan JH, Ismail HA, Cha GH, Hong SJ, Lee YH. Fasciola hepatica in snails collected from water-dropwort fields using PCR. Korean J Parasitol 2014; 52: 645-652.

9. Choi IW, Kim HY, Quan JH, Ryu JG, Sun R, Lee YH. Monitoring of Fasciola species contamination in water dropwort by cox1 mitochondrial and ITS-2 rDNA sequencing analysis. Korean J Parasitol 2015; 53: 641-645.

10. Sohn BW, Kang GS, Han TH. Studies on the optimal time for therapy of Fasciola spp. infected cattle in central area of Korea. Korean J Vet Serv 1992; 15: 1-6.

11. Cho SH, Lee CG, Kim JH. Laboratory maintaenance of field-collected Lymnaea viridis intermediate host for Fasciola hepatica. Korean J Vet Res 1997; 37: 185-188.

12. Novobilský A, Engström A, Sollenberg S, Gustafsson K, Morrison DA, Höglund J. Transmission patterns of Fasciola hepatica to ruminants in Sweden. Vet Parasitol 2014; 203, 276-286.

13. Caron Y, Martens K, Lempereur L, Saegerman C, Losson B. New insight in lymnaeid snails (Mollusca, Gastropoda) as intermediate hosts of Fasciola hepatica (Trematoda, Digenea) in Belgium and Luxembourg. Parasit Vectors 2014; 7: 66.

14. Rondelaud D, Vignoles P, Abrous M, Dreyfuss G. The definitive and intermediate hosts of Fasciola hepatica in the natural watercress beds in central France. Parasitol Res 2001; 87: 475-478. 